\title{
Les préfixes absolutifs de la première et de la deuxième personne singulier dans les formes marû ergatives*
}

\author{
Par Pascal Attinger - München
}

J. Krecher a, dans un article remarquable publié récemment ${ }^{1}$, émis l'hypothèse que les formes du type $(-) \mathrm{mu}+$ base marquent implicitement un absolutif de la lère ps. sg. («mich") ${ }^{2}$. Etant également arrivé, par des voies quelque peu différentes, à la conclusion que les éléments pronominaux de l'absolutif au singulier ${ }^{3}$ sont

* Abréviations: cf. en général l'auteur, ZA 74 (1984) 1 sq. Ajouter:

Edubb\& 1 = S. N. Kramer, Schooldays: A Sumerian Composition Relating to the Education of a Scribe, 1949 (aussi JAOS 69 [1949] 199-215) et nouveaux duplicats; $\mathrm{GiA}=\mathrm{W}$. H. Ph. Römer, Das sumerische Kurzepos 'Bilgameš und Akkar. AOAT 209/1 (1980); HF = M. Civil, The Home of the Fish. (. . .). Iraq 23 (1961) 154-175; Lú-digir-ra = id., The *Message of Lú-dingir-ra to his Mother» (. . .). JNES 23 (1964) 1-11; à compléter par M. Çı̆g/Kramer, The Ideal Mother: A Sumerian Portrait. Belleten 40/159 (1976) 413-421; Lullaby = Kramer/Th. Jacobsen, $u_{5}-a$ a-ù-a: A Sumerian Lullaby. Mél. Volterra VI (1969) 191-205; à compléter par B. Alster, RA 65 (1971) 170 sq.; RCU = P. Michalowski, The Royal Correspondence of Ur (Diss. Yale, 1976); SP = Sumerian Proverbs, cf. en général E. I. Gordon, BiOr. 17 (1960) 125 sqq. et 150 sq. et Alster, RA 72 (1978) 98-12. Noter en outre: abs. = absolutif; $\mathrm{B}=$ base verbale; $\mathrm{C}=$ consonne; loc.(-term.) $=$ locatif(-terminatif); ND = nom divin; $\mathrm{NP}=$ nom de personne; $\mathrm{pl} .=$ pluriel; $\mathrm{ps} .=$ personne; sg. $=$ singulier; $\mathrm{V}=$ voyelle.

1 Die /m/-Präfixe des sumerischen Verbums: Or. 54 (1985) 133-181.

2 Cf. surtout o.c. pp. 151 sq.: «Was schließlich ergativische mara-Formen angeht, 80 enthalten unsere Texte eine Reihe von Beispielen für /mu/- unmittelbar vor der verbalen Basis mit einem Ergativ der 2. oder 3. Person. Hier impliziert - oder darf man sagen: markiert? - /mu/- ein direktes Objekt der 1. Person/Singular («mich»). In ergativischen mara-Formen wird ja bekanntlich auch das direkte Objekt der 3. Person durch ein Personalpräfix vor der verbalen Basis markiert, also in einer Position, in der ein Personalpräfix sonst den Bezug auf den Ergativ (bei hamtu-Formen) oder auf ein dimensionales Komplement ausdrückt.»Cf. aussi p. 156.

3 Au pluriel, les exemples sont trop rares pour permettre de tirer des conclusions d'ordre général. Sur - ne - (préfixe abs. de la 3ème ps. pl.?), cf. C. Wilcke, cité par H. Hirsch/H. Hunger, AfO 28 [1981/2] 354 ad JCS 29, 186, et Krecher, o. c. 151, n. 38. 
préfixés dans les formes marû ergatives ${ }^{4}$, mais divergeant de Krecher quant à la structure morphologique de ces éléments, je présente, dans les pages qui suivent, ma propre interprétation des faits.

S. N. Kramer est, à ma connaissance, le premier à avoir noté (AS 10 [1938] 30 et n. 73, à propos de GEN 199) que - e- peut, dans les formes marni, représenter "the infixed accusative second person singular". Cette hypothèse resta toutefois sans écho jusqu'en 1970, où elle fut à nouveau envisagée par J. van Dijk (Or. 39 [1970] 308 et n. $1^{5}$; en dernier lieu Or. 52 [1983] 35 n. 11), qui ne réussit toutefois pas davantage à ébranler l'opinion courante, selon laquelle les éléments absolutifs de la lère et de la 2ème ps. sg. sont suffixés tant dans les bases marû que dans les bases hamtu (cf. en dernier lieu W. H. Ph. Römer, Einfühnung in die Sumerologie [1983] 57 sqq. et M.-L. Thomsen, The Sumerian Language. Mesopotamia 10 [1984] 147 sqq.).

L'hypothèse développée ci-dessous est que, dans les formes marâ (dont le point de substitution précédant la base n'est pas occupé par les éléments de l'ergatif), l'absolutif des lère et 2ème ps. sg., marqué par le morphème $\{e n\}$ (= "sujet" de la lère et de la 2ème ps. sg. des verbes non ergatifs), est placé immédiatement devant la base verbale. Au niveau graphique, \{en\} est soit explicitement noté (-Vn- à la lère sg., -e(n)-, après [i]: -in- à la 2ème sg. $\left.{ }^{6}\right)$, soit implicitement marqué par l'emploi des allomorphes $/ \mathrm{mu} /\left(\mathrm{non} / \mathrm{im} / /^{7}\right)$ de $\{\mathrm{m}\}$ et $/ \mathrm{ni} /\left(\right.$ non $\left./ \mathrm{n} / /^{8}\right)$ de $\left\{\mathrm{n}^{9}\right\}$.

4 Et probablement dans les formes hamfu cohortatives et impératives, mais je ne connais pas d'exemple sar. Pour ga-e-gi $i_{4}$, cf. ci-dessous p.172 et n. 34; pour ğar-mu-un, cf. ex. 88.

5 Apparemment indépendamment de Kramer, qu'il ne cite pas. Noter surtout: all semble que ces grammairiens étaient convaincus que - e- de la lère(?) et de la 2ème pers. était abrégé de: *-en-. Ensuite, qu'ils croyaient que l'accusatif de l'infixe ou du suffixe, de la lère et de la 2ème pers., était suffixé à des formes verbales de la lère et 2ème pers. prétérit du verbe transitif et qu'il était infixé aux formes correspondantes du présent. En quoi ils ont eu probablement raison. (. . .).» Cf. encore M. Yoshikawa, ASJ 4 (1982) 162 sq.

6 Sur cette distinction entre les allographes (et les allomorphes!) des profires de la lère et de la 2ème ps. 8g., cf. ci-dessous pp. $177 \mathrm{sq.}$

7 Cf. Krecher, o.c. 151 sq.

8 Ibid., passim; pour une interprétation légèrement différente, cf. l'auteur, ZA 74, 35, comm. de la 1.20.

9 Cette seconde possibilité semble être la seule envisagée par Krecher, l.c.; selon lui, la forme nam-mu-un-§ub-bé-en (ler millénaire) est fautive. Pour le préfixe abs. de la 2ème ps. sg., cf. toutefois p. 164, n. 69. 
Après avoir analysé un certain nombre d'exemples (groupés selon les périodes et les environnements préfixaux) et discuté succinctement les quelques données que l'on peut glâner dans les textes grammaticaux (OBGT et NBGT), j'essayerai d'interpréter les faits au niveau morphématique et synthétiserai, dans la conclusion, les résultats obtenus.

\section{Attestations ${ }^{10}$.}

I. absolutif de la lère ps. sg.

I. A. époque présarg. et époque d'Ur III: $\left\{\mathrm{m}^{11}+\mathrm{en}\right\}$ écrit $\mathrm{mu}$-. 1. Krecher, o.c. 152 et n. 41: Lugal/Nin-nam-mu-sub-bé/ e (NP) «Seigneur/Maîtresse, ne m'abandonne pas!"

2. Reisner, TUT 156 III 10': Nin- $\hat{g} u_{10}-\mathrm{mu}$-búr-e (NP) «Ma maîtresse me délivrera». De même $\mathrm{Mu}$ - $\mathrm{du}_{8}$-e (TUT $96 \mathrm{~V} 20$ et passim) et ${ }^{\mathrm{d}} \mathrm{Ba}-\mathrm{ba}_{6}-\mathrm{nu}-\mathrm{mu}-\S \mathrm{ub}-\mathrm{bé}$ (Th. Jacobsen, CTNMC 54 XI «25») (NP).

I. B. époque paléobabylonienne.

a) après $\{m\}:\{m+e n\}$ écrit: $(-) m u(-u n)-(e x .8-8)$

3. DD 95-97// 107-109:

$$
\text { mu(-un)-B-en(!) (ex. } 3 \text { et 8) }
$$

${ }^{*} u_{4}$ ki-zu ab-pà-dè-en-na-a ur-zu bu-mu-gu,-e (Q,a,b,c) $/ / \mathrm{hu}-\mathrm{mu}-\mathrm{lgu}_{7}-\mathrm{e}^{\mathrm{l}} \cdot \mathrm{en}(\mathrm{d})$

$u r-\hat{g} i_{6}$ ur-nam-sipa-da-zu

*ur-gi, ur-nam-en-na-zu ur-zu hgu-mu-gu ${ }_{7}-e(Q, a, b, c)$ $/ / \cdot \Gamma^{\top} e^{\top}(d)$

«Si je révèle (ton lieu =) là où tu es, puisse ton chien me dévorer, le chien noir, ton chien berger, le molosse, ton chien de «seigneurie», ton chien, puisse-t-il me dévorer!»

${ }^{10}$ La liste qui suit ne se veut pas exhaustive. Il convient par ailleurs de remarquer que les éléments /e/ et / $\mathbf{n}$ devant la base verbale étant polyvalents (/e/: ergatif de la 2ème ps. sg.; préf. du loc.-term.; /n/: ergatif/absolutif de la 3ème ps. sg. animée; préf. du loc.; etc.), un certain nombre des exemples cités peuvent être interprétés différemment. Je n'ai pas jugé opportun de rappeler, dans chaque cas, cette ambiguïté possible; elle doit toutefois rester constamment présente a l'esprit.

11 La structure syllabique des morphèmes en sumérien étant possible, mais non prouvée, je conserve la notation courante $\{m\}$ pour le ventif et $\{n\}$ pour le locatif. 
La forme hu-mu-gu ${ }_{7}(-e)-e n(d)$ est à rapprocher de mu-untúd-dè(-en) (Edubbâ 1, $17=$ ex. 8) et peut-être de h u - mu - un - ti le $(-e n)$ (ID $67+/ / 326^{12}$ ). Dans ces trois cas, la séquence préfixale (hu-) mu (-un)- + base marú semble n'avoir plus été comprise et pourvue (pléonastiquement) du suffixe -en, usuel dans les bases hamtu. (Avec l'abs. de la 2ème ps. sg., cf. ci-dessous ex. 21.)

4. $\mathrm{ELA}_{2} 61-63+/ / 92-94$ (cf. Krecher, o.c. 150, n. 36):

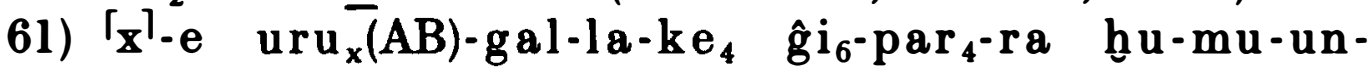
tú m- mu (A)

$$
\text { // hú -mu - }
$$

[x]-e ĝ $i_{6}-$ par $_{4}-\mathrm{ra}^{-k e_{4}}$ uru $\mathrm{u}_{\mathrm{x}}$-gal-la hu-mu(-un)-túm-mu [nam-l] ú-ùlu u $u_{6}$ du 10 $_{10}$ ge-es hu-mu-un-e (A)

$$
/ / \text { h } \mathrm{u}-\mathrm{mu} \mathbf{u}^{\mathrm{l}} \mathrm{e}(\mathrm{F})
$$

En 92 sqq.: ḩu-mu-e-túm-mu et ḩé-mu-e-e (formes verbales conservées seulement en $\mathrm{A}$ ).

«Puisse le [. .]. . de l'Urugal m'/t'amener dans le 'ĝipar', puisse le [. .]. . du 'gipar' $m$ '/t'amener dans l'Urugal, puissent les gens m'/t'admirer."

Cet exemple illustre bien l'opposition entre les préfixes de l'abs. de la lère et de la 2ème ps. sg. (-V)n- versus -e-). La forme $u_{6}$ du ${ }_{10}-g$ e-es hu-mu-un-e/ḩe-mu-e-e fait difficulté. L'explication la plus simple est d'y voir un exemple d' «attraction préfixalew ${ }^{13}$ (corriger en conséquence ma remarque en $\mathrm{ZA} 74,42$ ).

\section{ID 218-221:}

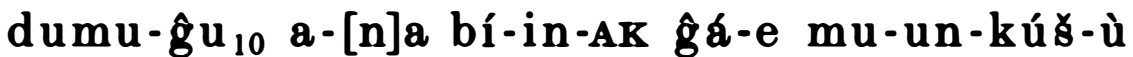

dinanna-k[e $e_{4}$ a-na] bí-in-AK ĝ́á-e mu-un-kús-ù (...)

"Qu'a donc encore fait mon (d'Enki) enfant! Moi, elle m'épuise! (...)."

6. UET 6, 295: hu-mu-un- gir $_{5}{ }^{-e} /$ hu-mu-u[n-s]ar-re /

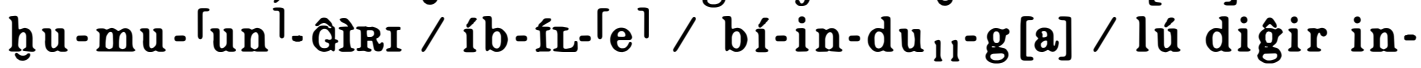
tuku-àm

12 Exemple peu clair, ma(-a)-ra (Emesal) faisant difficulté (datif éthique?).

13 Cf. par ex. SRT $6 / /$ 7, 85-88: a-a-ğ $u_{10}$ An lugal sipa (// a-a) diğir-re-e-

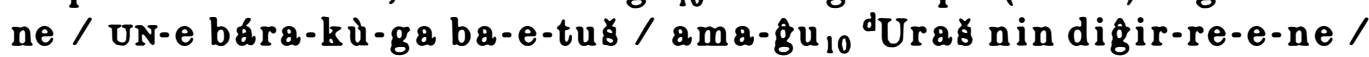
An-da ki-nú-kù-ga łà-kú §-ù e-ne-sù-ud gal ba-e-du ${ }_{11}$, kMon (de Ninisinna) père, An, le roi, le pattre (// le père) des dieux, s'assit publiquement(?) sur un trône étincelant. Ma mère, Urał, la maîtresse des dieux, ayant flirté avec lui, fut aimée par lui (grandement $\simeq$ ) comme jamais encore."

La séquence préfixale ba-e- n'est grammaticalement justifiée qu'à la 1.86 (préf. du loc.-term.). 
"Celui qui a dit: 'Qu'on me plonge (dans l'eau), qu'on me poursuive, qu'on me ..., je le (sup)porterai.', c'est un homme qui a un dieu (personnel).»

7. Cohen, Eræemma 63 sq., no 79, 11-17: (. . .) me-na mu-un$\mathrm{du}_{8}$-e (...), quand me délivrera-t-il (Nanna)/elle (Ningal?)?»" Cf. peut-être encore ex. 23, texte 0 .

8. Edubbâ 1, 17 (Ak = TLB 2, 7, I 3'):

$u_{4}$ na-ab-zal--e)-en um-mi-a-ĝt $u_{10}$ mu-un-túd-dè

// mu-un-túd-dè-en

$(\mathrm{A}, \mathrm{B})$

$/ /[\quad]^{\top} \mathbf{x}^{\top}$-GI.NE (Ak)

"Je ne dois pas m'attarder, sinon mon maître me frappera."

Sur la forme verbale de A et B, cf. ci-dessus ex. 3 et les lignes 26, 29 et 35-41: in-túd-dè-en, xil me frappa" (aussi texte C!). Contamination! ${ }^{15}$

Cf. encore Lugal-e 187; Juste souffrant $71(/ \mathrm{n} /$ : plus probablement préfixe loc.) et Sulgi A 93 (ex. 14). En Gilgames et Huwawa "149" (Kramer, JCS 1 [1947] 20), mu-un-bùlu $\hat{\mathrm{g}}$-e (D) // mu-e-

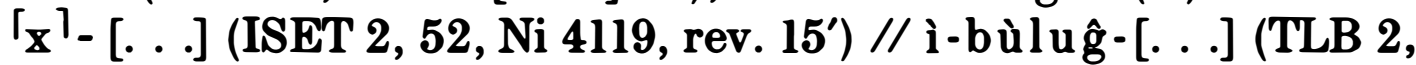
4,80 ) est probablement une forme hamtu.

b) après na-, (-)ba-: $\{n a / b a+e n\}$ écrit na/ba-an-

9. Instr. Sur. 118: na-an-§èr-§èr-re-dè (...) «Ne me lie pas!» Cf. ibid. 248: na-an-sèr-sèr-re-dè-en (-en = suffixe de l'ergatif, 2ème sg.!)

10. EnmEns. 256: nam-g ur ${ }_{4}-\mathrm{ra}-\mathrm{zu}$ ì-zu nam-ba-an-sis-edè-en "J'ai appris à connaître ta force, ne me 'rends pas amer'!" $(=$ "Ne rends pas mon sort amer!», v. s.)

11. SP 5.75:

A ur-gi ${ }_{7}$-re ĝ̀̀ri-pad-du

Q [ur]-gi, t̂̀iri-pad-du-gin nam-ba-an-sì-ge-dè-en "Chien, ne me malmène pas comme un os!" Sur la forme ur-gi ${ }_{7}-$ re de A, cf. SP $5.110^{16}$.

14 Cohen, o.c. pp. 64 sq., traduit: «When will she release her?" (le poete parle) et suppose (o.c. p. 63) que a ma - $\hat{g} u_{10}$ (l. 11) et a - a - $\hat{g} u_{10}$ (l. 15) sont des titres honorifiques, ce qui, vu le suffixe possessif, n'est guère vraisemblable (cf. l'auteur, ZA 74,36 ad 31).

15 Cf. inversément mu-un-túd-dè-en à la l. 26, texte B!

16 Sur le -e "vocatif», cf. en dernier lieu W. H. Ph. Römer, Zikir sumim = Mél. Kraus (1982) 312 ad 17, avec littérature antérieure (ajouter M. Civil, Le débat sumérien entre la houe et l'araire [1965] 83 sq.). 


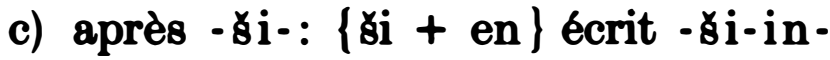

12. SRT $6 / / 7,108$ sq.: ki-bala kur saĝ-ki gíd-da-ni-sè / a-a- $\hat{g} u_{10}{ }^{d} E n-l i ́ l-l e ~ i m-m a-s i-i n-g i_{4}-g i_{4}$ *Vers le pays révolté, le pays ennemi qu'il a regardé en fronçant les sourcils, mon père Enlil m'envoie.»

13. EnmEns. 53 sq.: za-e-sè lugal-ĝgu $u_{10}$ mu-e-si-in-gi ${ }_{4}-$ nam (B) // mu-e- $§ i-i n-g i_{4}-g i_{4}$ (D) / en-Arattaki En-suhkesda-an-na-ke ${ }_{4}(\ldots)^{17}$

«Mon seigneur/Ensuhkešdanna, le seigneur d'Aratta, m'a envoyé vers toi." (ainsi B; c'est la formule traditionnelle, cf. par ex. ELA 176 sq.; 185; 379 et 515 sq.); D: «Mon seigneur (. . .) m'envoie vers toi."

d) après $\{n\}:\{n+e n\}$ écrit: $-n i(-i n)-(e x .14)$

$$
\text { -e-ni-(?) (ex. 15) }
$$

14. Sulgi A 93 sq.:

š̀r-kù-ĝ́á hà-mu-un-è(-e)-ne (A, O, R, PP, SS(?))

$/ /$ hu-mu-un- $\Gamma^{\top}-$ ne (B)

$/ /$ hu-mu(-un)-ne-è/e $11-n e\left(C\right.$, II, TT) ${ }^{18}$

nam-mah-ĝgu $u_{10}$ huu-mu-ni-in-pd-dè-ne (A, B, PP, II(?))

$/ /-\hat{g} a ́$ (PP) // hu-mu-ni-pà-dè(-en)-ne $(C, 0)^{19}$

"Puissent-ils m'exalter ${ }^{20}$ dans un pur chant en mon honneur, puissent-ils ( $m$ 'y révéler vers ma grandeur $=$ ) m'y révéler dans ma grandeur." (conjectural)

15. SP 1.4-5 (cf. W. G. Lambert, BWL 262 et B. Landsberger, AfO Bh. 17 [1967] 33 sq.): 'Nin-ĝz-zi-da-ra ga-ti na-an-naab-bé-en / ká-na nam-mu-e-ni-dib-bét

Soit: «Ne dis pas à Ninğizzida: 'Je veux vivre!', mais: 'Ne me fais pas passer par (ta) porte!'.", soit: «Ne dis pas (. . .), mais qu'il ne te fasse pas passer par (sa) porte." $=$ "Ne dis pas a $\mathrm{N}$. que tu veux vivre, mais (demande-lui) de ne pas te faire passer par (sa) porte."

17 DD épigraphiquement incertain, probablement [mu-e-si-i]n-gi $-i[n-n a m]$.

18 = probablement /bumunene/, non /bumune'ene/!

$19 \mathrm{R}, \mathrm{RR}$ et TT semblent corrompus.

20 La forme mara e/e $e_{11}$ (au lieu de $e / e_{11}-d$ ) est inattendue. "Jeu de mots" sur sirku $\mathrm{e}$, "(dire $=$ ) chanter un pur chant"?

21 La forme nam-mu-ni-íb-dib-bé-en-zé-en(-e-8e) (= acc. e tu-us-bi-a-ni[in-ni-mi]) de BM 38283, 10 sq. (BWL 262) reste obscure. 
La première possibilité semble plus naturelle (cf. aussi la traduction accadienne citée dans la n. 21); ce serait toutefois le seul cas où - e- marquerait le préf. abs. de la lère ps. sg. ${ }^{22}$

I. C. époque postpaléobabylonienne.

Cf. par ex. a-ba mu-un-รed $\mathrm{d}_{7}-\mathrm{dè}=$ man-nu ú-na-ah-ha-an-ni, "Qui peut m'apaiser?" (Cohen, Eršemma 139, no 10, 1) et nammu-u n-sub-bé-en = la ta-nam-da-an-ni, "Ne m'abandonne pas!" (ibid. 29 sq., no 29, 1-21).

Résumé de I: A de rares exceptions près (ex. 3, texte d; 8 , textes $A$ et $B$ et $\mathrm{D}$ 67, texte $C_{1}{ }^{23}$ ), le morphème de l'abs. de la lère ps. sg. est préfixé dans les bases mará. A l'époque présarg. et à l'époque d'Ur III, il est implicitement marqué par l'allomorphe /mu/ de $\{\mathrm{m}\}$; à partir de l'époque paléobab., il est normalement explicitement noté (graphie: -Vn-), plus rarement implicitement marqué par l'emploi des allomorphes $/ \mathrm{mu} /$ et $/ \mathrm{ni} / \mathrm{de}\{\mathrm{m}\}$ et de $\{\mathrm{n}\}$. Dans un cas (ex. 15), il est peut-être noté par -e- (confusion lère/ 2ème ps.!).

\section{II. absolutif de la 2 ème ps. sg.}

II. A. époque présargonique: $\{\mathrm{ba}+\mathrm{en}\}$ écrit ba- $(\{\mathrm{en}\}$ non explicité).

16. Instr. Sur. 19 (cf. C. Wilcke, ZA 68 [1978] 213 sq.):

$L$ (n.p.) §u-du $u_{8}-a$ nu-e-tùm lú-bi $\quad a-b[a-\ldots .$.

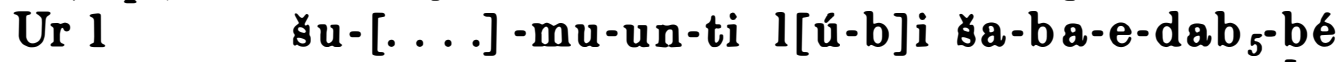

T 1 ] ]-tùm lú-bi se-ba-e-dab ${ }_{5}^{-}{ }^{-b_{b}}{ }^{\top}$

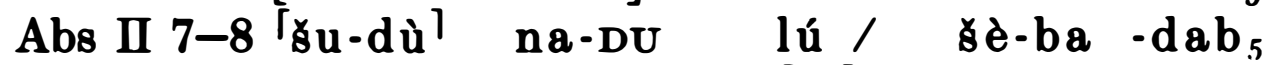

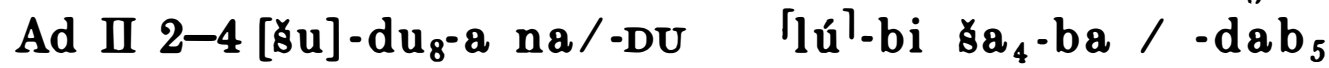

"N'apporte pas de garant, cet homme se saisira de toi."

L'abs. de la 2ème ps. sg. n'est explicité que dans les textes paléobab.

22 Sur la séquence -e-ni- (préf. de l'abs. 2ème ps. sg. + préf. loc.), cf. ci-dessous ex. 29 et n. 36; sur l'origine de cette séquence, cf. n. 46.

${ }^{23}$ Noter qu'à l'exception de DD 95 // texte d, ces formes ne se laissent pas davantage expliquer dans le cadre de la théorie "classique" (suffixation des morphèmes de l'abs. de la lère et de la 2ème ps. sg. dans les bases mará également), le - n devant la base faisant alors difficulte; cf. aussi n. 40. 
II. B. époque de Gudea: $\{\mathrm{n}+\mathrm{en}\}$ écrit - $\mathrm{ni}$ -

17. Cyl. B II 21 sq.: dNin-ĝ́r-su é-zu ma-ra-dù / húl-「la $\mathrm{ha}-\mathrm{ni}^{\mathrm{l}}-\mathrm{ku}_{4}-\mathrm{ku}_{4}$

"Ningirsu, j'ai bâti ton temple, puissé-je ${ }^{24}$ t'y faire entrer dans la joie."

La traduction proposée par A. Falkenstein (SAHG 167): «(. . .) In Freude mögest du dort einziehen!" ne rend pas compte de l'allomorphe /ni/ de \{n\}. Au niveau sémantique, cf. Cyl. B II 5: sipa me é mu-dù lugal-ĝu $u_{10}$ é-a-na mi-ni- $\mathrm{ku}{ }_{4}-\mathrm{ku}_{4}$, "Moi, le pâtre, j'ai construit le temple, je vais faire entrer mon seigneur dans sa maison." et ibid. V l: ur-sa ĝ ${ }^{d} \mathrm{Nin- \hat {g } i ́ r - s u ~ e ́ - a ~ m i - n i - ~}$ $k u_{4}-k u_{4}$, "Il fait entrer le héros Ningirsu dans le temple."

II. C. époque d'Ur III: [a] + \{en\} écrit -(C)a(-an) -

18. H. Limet, L'Anthroponymie sumérienne (1968) 321 sq. (cf. D. O. Edzard, BiOr. 28 [1971] 164):

Les NP Ha-ba-zi-zi (TUT 100 I 9; etc.), Ha-ba-an-zi-zi (ibid. 125 IV 16; etc.), Ha/Hुé-ma-zi-zi (UDT 69, 33; etc.) et Ha-ma-an-zi-zi (HSS 4, 47, 16'; etc.) peuvent difficilement être détachés du type (ND-e-)ba(-an)-zi-ge(-en) (par ex. dNinlíl-e-ba-zi-ge; graphie non abrégée du suffixe en CT 32, 45, BM 103428,2: Ba-zi-ge-en $\left.{ }_{6}\right)^{25}$. La seule interprétation rendant compte de toutes les formes est de voir en -an-/-e(n) le préfixe/suffixe de l'abs. de la 2ème ps. sg. Traduire par conséquent:

Ha-ba(-an)-zi-zi *Puisse-t-il (le dieu) te (= l'enfant) faire te lever ici!»

Ha-ma(-an)-zi-zi "Puisse-t-il te faire te lever pour moi!» (ND-e-)ba(-an)-zi-ge(-en) «ND/Il t'a fait te lever ici.»

24 Sur bé/ba/bu - (précatif) + lère ps. sg., cf. par ex. Lugal-e 128; EnmEns. 148 // 161(?) (cf. l'auteur, RA 78 [1984] 110 et n. 52); GiA 54 (traduire: «Puissé-je faire se lever un brave!»); RCU 21, 17 (traduire: «Puissé-je m'emparer de Kazallu!»; $\widehat{\gamma} u-\hat{g} u_{10} / \hat{g} a ́$ est un instrumental); Isin *18, 11 (ex. 24); etc.

25 Edzard, l.c., sépare la série des NP en -zi-zi (Ha-ba/ma(-an)-zi-zi = «er möge ihn dort/mir aufrichten*) de celle en-zi-g (*nin-e-ba-n-zig-e(n) = «die Herrin hat mich/dich 'abgebucht'», métaphore empruntée au vocabulaire de la vie économique). 
II. D. époque paléobabylonienne.

a) après $\{m\}:\{m+e n\}$ écrit: $(-) \mathrm{mu}-\mathrm{e}^{26} /(-) \mathrm{me}(-\mathrm{e}) \cdot(\mathrm{ex} .19-21)$ me-en- (ex. 22) mu-un-(?) (ex. 23 et lugal-e 552)

$(-) \mathrm{mu}-\mathrm{u}_{8^{-}}($ex. 24)

19. Lullaby $49 \mathrm{sq}$ :

ù-a-lum-e ${ }^{a}$ h̆é-me-lum-lum-e / ù-a-lam-e b hé-me-lamlam-e

a) // ̀̀-a-lum-lum // ù-a-lum-ma b) // ù-a-lam-lam // ù $-\mathbf{a}-\mathbf{l} \mathbf{a} \mathbf{m}-\mathbf{m} \mathbf{a}$

"Puisse l' 'ualum(lum)' te faire prospérer, puisse l'ualam(lam)' te faire exubérer!» ${ }^{27}$

La non-explicitation de l'ergatif dans trois textes sur quatre n'est pas surprenante après une interjection.

20. Instr. Sur. 246: níg nam-kal-kal-en níg-e me-kal-e (U 2) // mu- $\mathrm{e}^{\top}$ - [...] (C 3)

"N'estime pas trop (kal-kal!) les choses, c'est aux choses à t'estimer." (C'est-à-dire: La richesse dépend davantage du sort que de l'effort personnel pour les acquérir.) $)^{28}$

21. Lugal-e 426:

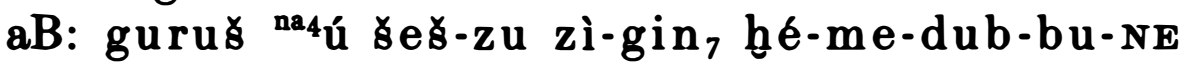

$\mathrm{d}_{1}:{ }^{\text {na4}}{ }^{\mathrm{u}-a}$ sez-a zì-gin, hé-mu-e-dub-bu-dè-en sam-mu ŠS.MES-ka ki-ma zì.DA lit-bu-ku-ka

aB: weune homme, pierre-plante, que ton/tes(?) frère(s) te répande(nt) comme de la farine!"

$d_{1}$, sum.: "Pierre-plante, que l'on te répande parmi (tes) frères comme de la farine!"

acc.: «Pierre-plante, que tes frères te répandent comme de la farine!»

La forme hé-mu-e-dub-bu-dè-en semble être pourvue (pléonastiquement) du suffixe -en (sur ces formes hybrides, cf. encore ex. 25, 29 et 30; avec l'abs. de la lère ps. sg., cf. ci-dessus ex. 3).

${ }^{26}$ La lecture me $e_{x}$ - pour MU.E-, proposée par Krecher, o.c. $164^{69}$, est possible, mais reste, en l'absence de données lexicales, difficilement prouvable.

27 Sur lum/lam, cf. en dernier lieu G. Farber-Flügge, Studia Pohl 10 (1973) 233 sq. Traduire peut-être «l' $u a^{\prime}$ qui fait prospérer/exubérer", v. 8.

${ }^{28}$ Ou peut-être: «N'estime pas trop les choses, et (les choses te feront estimer =) l'on t'estimera pour ce que tu as." 
De même: Lugal-e 424 (aB: h̆é-me-zi/zé-ir-zi/zé-ir-re-dè; $\mathrm{d}_{1}$ : hấ-en-zi-re-dè $\left.=l i-p a-s i s-k a\right) ; 471$ sq. (hé-mu-e-zi-zi-ne

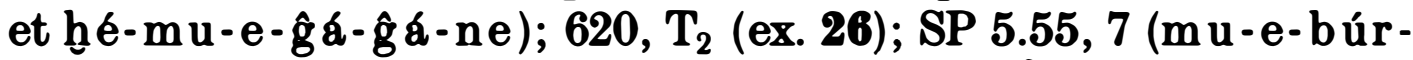
e-en, ije te laisse allerw(?)) et les ex. 22-24 ${ }^{29}$.

22. Lugal-e 539 sq.:

ki-enim-ma-ka (im-) mu-e-ni-bulu $\hat{g}_{3 / 5}-\mathrm{en}$

ad-gi ${ }_{4}-g i_{4}-d a$ gá-e me-en-bùluĝg-en $\left(J_{2}, G_{2}(?)\right)$

$$
\begin{aligned}
& / / \mathrm{mu}-\mathrm{e}-[\mathrm{I} \\
& / / \mathrm{mi}-\mathrm{ni}-2 b-b u l u \mathrm{~g}_{5}{ }^{-} \mathrm{K}_{\mathbf{x}}{ }^{1}\left(\mathrm{H}_{2}\right)^{30}
\end{aligned}
$$

"Tu m'as vanté au tribunal, moi, je te vanterai à la délibération."

C'est, à ma connaissance, le seul cas où $\{e n\}$ est écrit - en- après $\{\mathrm{m}\}$.

23. Nm\& 75 sq. (FF = ISET 1,190 , Ni 9862, rev. $2^{\prime}$ sq.; $P P=U M$ 29-15-523, A. J. Ferrara, JCS 28 [1976] 96, no 94, rev. 10' sq.; QQ $=$ UM 29-13-513, ibid. 96 sq., no 95, II 17' sq.; YY = SLFN 32, 3NT 905, 184, 4' sq.; GGG = ibid. 35, 3N-T 917, 393, $9^{\prime}$; HHH = ibid. $32,3 N-T$ 917, 401, rev. 2' sq.; cf. Wilcke, WZKM 68 [1976] 88 et Römer, UF 4 [1972] 186 sq., qui interprètent le passage différem-

\begin{tabular}{|c|c|c|c|c|c|c|c|c|}
\hline 75) & An-ra & $d u_{11}-m$ & $m u-1$ & $n a-\varepsilon$ & & An- $\mathbf{e}$ & ba-ma. & $-d u_{8} \cdot e$ \\
\hline $\mathbf{A X}$ & $-\quad-$ & - & - & - & - & $-\quad-$ & $-\quad-$ & $-\quad-$ \\
\hline $\mathrm{C}+$ & - & - & - & - & - & - & - & en \\
\hline D & - & enim & - & - & -bé & né & - & en \\
\hline $\mathbf{J}$ & - & - & - & - & - & - & - & - \\
\hline 0 & - & - & - & - & - & né & - & - \\
\hline$U_{1}$ & - & - & - & - & - & né & - & - \\
\hline $\mathrm{U}_{3}$ & lú $t$ & & & & & & & ] \\
\hline $\begin{array}{l}\mathrm{U}_{4} \\
\mathrm{FF}\end{array}$ & $-\bar{f}$ & $t$ & 子 & - & [ & & & ] \\
\hline PP & {$\left[\begin{array}{l}\tau \\
{[}\end{array}\right.$} & & 子 & - & - & - & ba & $\mathrm{r}_{\mathbf{x}}$ \\
\hline QQ & {[} & & 子 & - & $-b[e$ & & & ] \\
\hline YY & [ & 子 & - & - & - & né & $t$ & ] \\
\hline $\begin{array}{l}\text { GGG om. } \\
\text { HHH }\end{array}$ & $-r$ & & & & & & & I \\
\hline
\end{tabular}
ment.):

29 En HF 149 (cf. $138 / /$ ) -e-dans nu-mu-e-lá-e et nu-mu-e-NE.NE est plus probablement le préf. du loc.-term.

30 Forme corrompue? Texte d'Ur! 


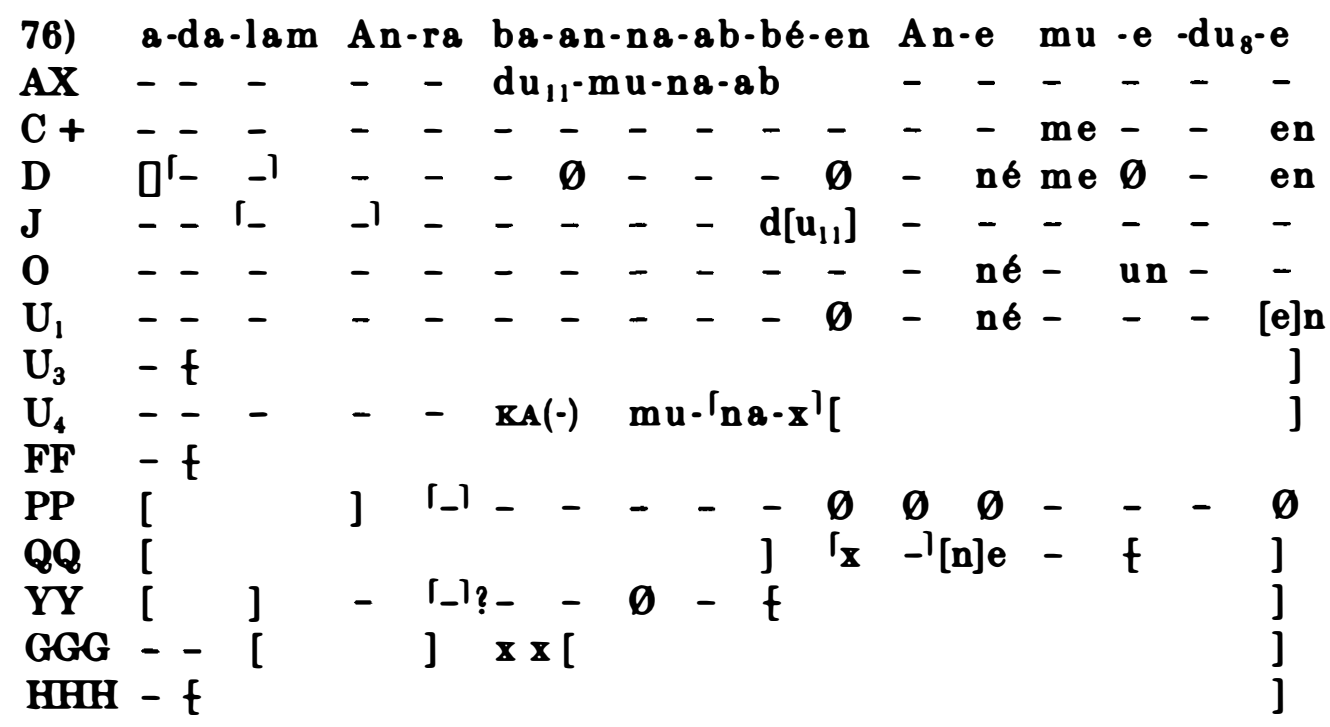

J'admets que la 1.75 est une prière d'Enheduanna à Inanna, et la 1. 76 la réponse d'Inanna. Dans les lignes qui suivent, Enḩeduanna exalte Inanna (à la 3ème ps.), puis s'adresse de nouveau directement à elle (83):

«- Dis-le (mon destin) à An, puisse An (le) dénouer pour moi (ha ma-du $\mathbf{8}^{-e}$ ) // puisses-tu le faire dénouer pour moi par An (ba$\left.m a-d u_{8}-e n\right)^{31}$.

- Je vais le dire dès maintenant à An, et An va te délivrer (mu -

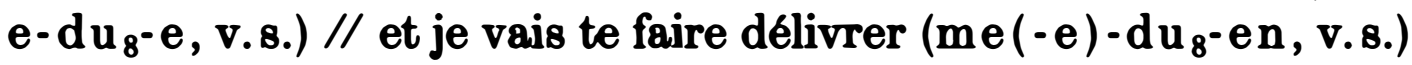
par An."

Si l'on prend au sérieux la variante de $0\left(m u-u n-d u_{8}-e\right)$, il faut soit traduire: "(...) Tu vas le dire a An, et An va me délivrer." (sans dialogue), soit admettre que l'abs. de la 2ème ps. sg. peut être, exceptionnellement, explicité graphiquement par -Vn- également après /mu/ (après (-)Ce/i-, c'est la graphie usuelle à côté de Ø).

24. Isin *18, $11 / / 28+/ / 13 / / 30$ (cf. Römer, SKIZ 21, 10 et W. W. Hallo, BiOr. 23 [1 $\overline{966}]$ 244, $10 / /$ (B seulement en transcription)):

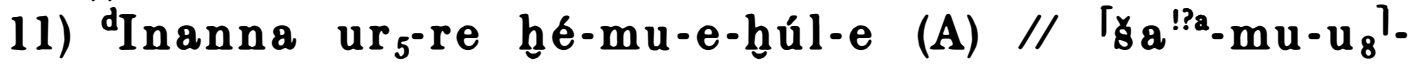
húl-1[e]

28) b dnanna ur $r_{5}$-re sa-mu- $u_{8}$-húl-le

13) // $30^{b}{ }^{d}$ Nin-é-gal ur $_{5}$-re $8 a^{a}-m u-u_{8}-h u_{u} l-l e$

31 L'ergatif second dans une construction causative est normalement marqué par le datif, mais le (loc.-)term. est parfois atteste, et peut s'expliquer, dans notre passage, par le souci d'éviter la répétition de An-ra. 
a) Aux ll. 11 et 13 (mais non 28 et 30!), B porte apparemment: ga-mu- $u_{8}-h u ́ l-l e$, ce qui est certainement une faute du scribe.

b) $\mathrm{A}$ om.

"Inanna/Ninegal, puissé-je ainsi te réjouir!" ou "(. . .) puisse cela te réjouir!» Cf. l. 17: 'Inanna š̀-zu hué-em- hhúl'-[le] (B) // ḩému-e-ḩúl-le (A), "Inanna, puisse ton coeur se réjouir de cela!» En $A$, probablement *attraction préfixale ${ }^{32}$.

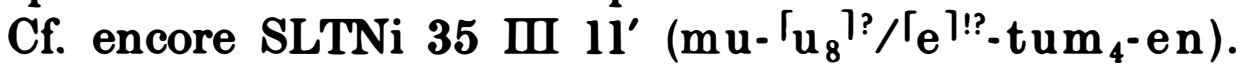

b) après ba-: $\{b a+e n\}$ écrit: ba-e- (ex. 16 et 25)

$$
\text { ba-e-B-en(!) (ex. 25, Z) }
$$

25. GEN 199 (cf. 221): i-dUtu kur-ra ba-e-dab ${ }^{-b e ́ ~(r, ~ A A) ~}$ $/ / b_{a}-\Gamma^{\top}-d_{a b}-b e ́-e[n](Z)$ "Le 'Hélas, Utu!' te saisira dans le 'kur'."

Cf. ibid. 221: i-dUtu kur-ra/re im-ma-an-dab 5

Le - $n$ - devant la base n'est pas sans faire difficulté (i- ${ }^{d} U$ tu personnalisé? $\mathrm{Ou} / \mathrm{n} /=$ morphème de l'abs. de la 3ème ps. sg. animée, préfixé dans une forme hamțu?!); y voir l'allomorphe du préf. loc. $\{\mathrm{n}\}$ (au lieu de /ni/ attendu) n'est toutefois guère vraisemblable, la séquence im-ma-ni-in- étant bien attestée aux ll. 211; 213; 216 et $218-220^{33}$. Le -e- de ba-e-dab $b_{5}$ bé ne peut être, en conséquence, le préf. du loc.-term. Sur ba- $\int^{\top}-d a b_{5}-b e ́-e[n]$, cf. ci-dessus ex. 21.

Cf. encore ex. 16. En EnmEns. 268 (R: dNanna lugal- ‘tùr 1 .

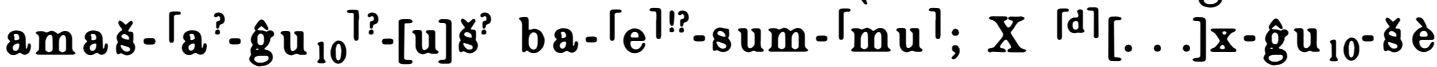
ga(-)ba-e-sum-mu) et HF $138 / /($ su- zè ba-e-lá-e), -e-est plus probablement le pref. du loc.-term. (avec non-explicitation graphique du préf. de l'abs. 2ème sg.).

c) après ga-: pas d'exemple sûr.

En Enlil Sud 29 (ga-e-gi ${ }_{4}$ Eres ${ }^{k i}$ uru ${ }^{d} \mathrm{Nisaba-sè)} \mathrm{et} \mathrm{Lú-}$

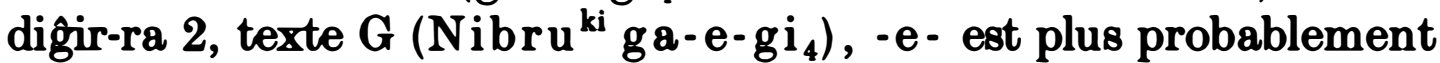
le préf. du loc.-term. (cf. Enki Ninmah 95: ba-e-gi $\left.{ }_{4} !\right)^{34}$.

Sur "ga-mu-u $u_{8}-h u ́ l-l e *$, cf. ci-dessus ex. 24, n.a.

32 -e- ne peut être le préf. du loc.-term., hu úl = «se réjouir de qqc." se construisant avec le préf. du terminatif (syntagme nominal: terminatif, plus rarement loc.term.).

${ }^{33}$ Cf. par ailleurs GE XII $27 \pm / / 46: t a-z i-i m-t i / t a-a z-z i-i m-t u m \mathrm{KI}^{\text {tim }} i$-sa-bat-ka \pm // ta-z[i-im]-ti $\mathrm{kI}^{\text {tim }}$ is-sa-bat-s[u].

34 Remarquer toutefois que la forme n'est pas suffixée ( ${ }^{*} g a-e-g i_{4}-i n$, v. 8.$)$, et qu'elle doit être, par conséquent, morphématiquement analysée en: $\{g a+e+e n$ 
d) après bí-, (-)mi-: bí-/(-)mi- + \{en\} écrit bí-/(-)mi $(-$ in $)$ -

26. Lugal-e 620:

aB: kur-kur-re nar-gin, gi di-da há-em-mi-in-sar-ne

$$
\begin{array}{r}
\left(\mathrm{S}_{2}(?), \mathrm{U}_{2}\right) \\
/ /[\mathrm{h}] \text { é-em-mu-e- }-\Gamma_{\mathrm{s} \text { ar }}{ }_{-} \\
\text {re-ne }\left(\mathrm{T}_{2}\right)
\end{array}
$$

$n_{1}: k u[r-r]$ e $[n]$ ar gi $\left\lceil^{\left[d i-x-x^{\top}\right.}\right.$

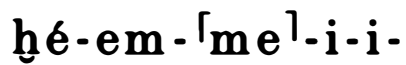

$n e^{?}($ fB)

acc.: trop cassé pour être utilisable.

aB: «Puissent tous les pays, tel un musicien, te faire tournoyer au (son de) la flate!".

$\mathrm{n}_{1}$ : «Puissent les pays, tel un flatiste, te louer!»

Cet exemple illustre bien la distribution des allographes de $\{e n\}$ : - $\mathrm{mu}$ - $e$ - versus $-\mathrm{mi}-\mathrm{in}$ -

27. Sjöberg, Mél. Finkelstein (1977) 191, 81 sq.: i-bí-zi-íl-la-za mu-ni-in-pa-dè mu-pa-da-ĝgu $\mathbf{u}_{10}$-me-en An-gal-e kur zà-til-la-ba sù-ud-šè bí-in-diri-ge "Parce que tu as fidèlement levé les yeux (vers moi), je te choisis, tu ess mon élu. Le grand An te rendra à jamais supérieur (a tous) jusqu'aux confins des pays."

A la 1. 82, une forme hamţu, sans être exclue, est moins probable, vu le parallélisme avec la 1.81 et la graphie - ge, non - ge-en (cf. l. 89: $\mathrm{mu}-\mathrm{u} n-p[\mathrm{a}-\mathrm{d}] \mathrm{è-en!})^{35}$.

e) après $\{n\}:\{n+e n\}$ écrit: -ni-in- (ex. 27-29)

$$
\begin{aligned}
& -e-n i(-\operatorname{in}(!))-(\text { ex. 15(?); } 29 \text { et } \\
& \text { DD 51) } \\
& -n i-i n-B-e n(!) \text { (ex. 30) }
\end{aligned}
$$

28. Sjöberg, StOr. 46 (1975) 306, 12'// 26'// [33']: en ${ }^{d} \mathrm{Nin}$ ĝiz-zi-da zà-mí-zu du ${ }_{10}$-ga s̀̀r-re-es àm-mi(-ni)-in-ne "Seigneur Ninĝizzida, il est doux de te louer, je/on t'invoque dans des chants." -ni-in-ne $=\{n+e n\}+$ base e.

$+B\}$, avec non-explicitation graphique de $\{(n\}$. Un contre-exemple possible est EnmEns. 268, X: ga (-) ba-e-su m-mu (=\{ga + ba + e + B + en $\}$ ?), mais la ligne est épigraphiquement peu sûre.

${ }^{35} \mathrm{mi}$-ni-in-pa-dè (81) ne peut être invoqué comme un contre-argument, l'abrégement de -en en -e étant fréquent avec l'ergatif lère/2ème sg. 
29. DD 55:

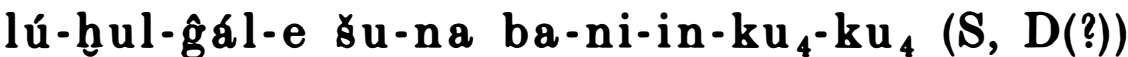

$$
\begin{aligned}
& / / \text { ba- } \mathrm{re}-\mathrm{ni}{ }^{1}-[\ldots \ldots](\mathrm{R}) \\
& / /{ }^{*} \mathrm{ba}-\mathrm{e}-\mathrm{ni}-\mathrm{in}-\mathrm{ku} \mathrm{u}_{4}-\mathrm{ku}_{4}(\mathrm{O}, \mathrm{P}(?))
\end{aligned}
$$

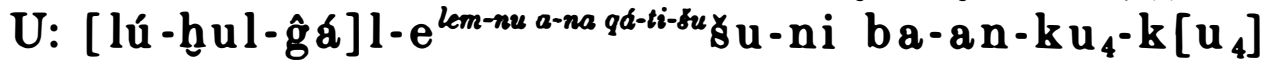
"Le méchant (te fera entrer dans sa main =) mettra la main sur toi."

La séquence préfixale $-\mathrm{e}-\mathrm{ni}^{36}$ représente normalement, au niveau morphématique, $\{n+e \text { (ergatif?) }\}^{37}$, ici toutefois $\{n+e n\}$. Sur la forme hybride ${ }^{*} b a-e-n i-i n-k u_{4}-k u_{4}$, cf. ci-dessus ex. 21.

Cf. encore DD 51 (lire ní mu(-e)/me-ni-dab $b_{5}$-bé!).

30. ISET 2, 8-10, Ni 9721+, IV 27 + // IV 30 (Inanna et Sukalletuda; cf. Kramer, ArOr. 17/1 [1949] 402 sq., n. 16 et D. O. Edzard, ZDMG 109 [1959] 239 sq., n. 12a): munus-e [§] à-kurkur-ra-ka nu-um-ma-ni-in-pa [d]è-en

"La femme (= Inanna) ne te découvrira pas au coeur des pays étrangers.»; cf. IV 30: [munus-e s̀-kur-kur]-ra-ka nu-umna-ni-in-[p]d. Forme hybride!

f) après hấ-: \{ha + en\} écrit: hgé- (passim) h̆é-en- (ex. 31)

31. Lugal-e 577 (pour $\mathrm{Y}_{3}+\mathrm{E}_{5}$, cf. M. J. Geller, BSOAS 48/2 [1985] 221):

${ }^{\text {*na4 }}$ mał-da udu-gin, haé-en-SUR.SUR-ne $\left(Y_{3}+E_{5}\right)$

// hé-sur.sur-ne $\left(\mathrm{O}_{2}\right)^{38}$

"Pierre-gazelle, qu'ils/qu'on te ... ${ }^{39}$ comme un mouton!"

Sans explicitation graphique de l'abs. 2ème sg., cf. Lugal-e 455 sq. (hé-sĩ-ge et hue-gul-e); 556 (aB: h̆[é-ga]z-[e]// ḩé(-en)gaz-gaz dans les textes postpaléobab.) et 579 (hé-AK-ne).

\section{E. époque postpaléobab.}

Les règles orthographiques de l'époque paléobab. restent valables, mais les formes "fautives" se multiplient.

${ }^{36}$ Cf. en général M. Yoshikawa, ASJ 4 (1982) 153-169, et, en dernier lieu, Krecher, o.c. 164, n. 69 ; cf. aussi ci-dessous n. 46.

$37 \mathrm{Cf}$ u-mu-e/me-ni- dans les incantations.

${ }^{38} \mathrm{~F}_{1}$ et $\mathrm{N}_{2}$ sont épigraphiquement peu clairs.

39 Cf. Sjöberg, Der Mondgott Nanna-Suen (1960) 13, 5 (commentaire pp. 19; 33, n.

7) et W. Heimpel, Studia Pohl 2 (1968) 108. 
après $\{m\}$ : cf. ex. 21; 26 et Lugal-e 471 sq. (ḩé-mu-e-[...]). après haé-: cf. Lugal-e 424 (hé-en-zi-re-dè = li-pa-sis-ka); 553 (hé-tag-ga $=l i-s a l-l i t-k a)$ et 556 (hée(-en)-gaz-gaz $=l i-i h /$ lih-süu-ul-ka).

Résumé de II : A quelques exceptions près (ex. $21 \mathrm{~d}_{1}, 225, \mathrm{Z}$ et $30)^{40}$, le morphème de l'abs. de la 2 ème ps. sg. est préfixé dans les bases marî. [a].

- A l'époque présarg., il est graphiquement non explicité après

- En Gudea, Cyl. B II 22 (ex. 17), il est implicitement marqué par l'allomorphe /ni/ de $\{n\}$.

- A l'époque d'Ur $I I$, la séquence - Ca- + \{en $\}$ est notée -Ca (-an)- (versus -Ca-e-à l'époque paléobab.).

:- A partir de l'époque paléobab., il est normalement explicitement noté, si ce n'est après hé -. La distribution des allographes est claire:

précédé de $[u]$ et de [a]: -e - (mu-e- souvent écrit me (-e)-, une fois - mu- $u_{8}-(e x .24)$, une fois me-en- (ex. 22)).

précédé de [i]: -in- (dans deux cas, $\{n+e n\}$ écrit -e-ni-). précédé de [e]: -en-.

\section{Données lexicales (OBGT et NGBT) ${ }^{41}$.}

Les rares renseignements que l'on peut glâner dans les textes grammaticaux paléobab. et néobab. sont malheureusement peu concluants.

OBGT

III. A. préfixe de l'absolutif de la lère ps. sg.

32. OGBT III, 232 sq. (cf. Black, o.c. 15):

$\mathrm{mu}(-\mathrm{un})-\mathrm{ti}-\mathrm{ti}=\dot{u}-r e-\xi e-a n-x i$ wil me traite durement" $\mathrm{nu}-\mathrm{mu}(-\mathrm{un})-\mathrm{ti}-\mathrm{ti}=\dot{u}-l a$ ú-re-[รe-an-ni]

40 Ces formes sont anomales également dans le cadre de la théorie classique; cf. $n$. 23.

41 Cf. en général J. A. Black, Sumerian Grammar in Babylonian Theory. StPohl SM 12 (1984). 
Formes correctes! En revanche, ma-te-te = tur-tas-sa-an-ni («Berlin Vocabulary" (néoass.!) I 24; cf. Reisner, ZA 9 [1894] 159) ne m'est pas clair.

33. ibid. VI 46 (cf. Black, o.c. 95):

gar-mu-un = su-uk-na-an-ni "place-moi!»

Mais cf. ibid. VI 5l: [ $\hat{\mathrm{g} a r}$ ]-mu-ub $=\xi u-u \xi ̌-k i-n a-a n-n i(-\mathrm{n}$ succombe devant le $-b$ ncausatifw).

III. B. préfixe de l'abs. de la 2ème ps. sg.

34. ibid. IX, 98-104.

\begin{tabular}{|c|c|}
\hline $\begin{array}{l}\int_{s} a^{l} \text { àm-e } \\
\text { sá àm-e-en } \\
\text { sá àm-e-en }\end{array}$ & $\begin{array}{l}i-k a-a \xi-s a-d a m \\
\text { (akałsadam) } \\
\text { (takałళadam) }\end{array}$ \\
\hline 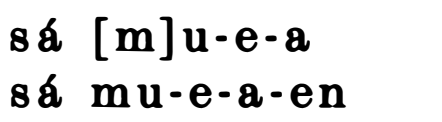 & $\begin{array}{l}i-k a-a s_{-}-s a-d a-k a \\
\text { (akałsadakka) }\end{array}$ \\
\hline$a-e$ & (aktasdakka) \\
\hline
\end{tabular}

Cette série de formes se laisse interpréter logiquement si l'on admet: 1) que -e-a (-en) $<*$-e-e $(-$ en $)=\{e n+$ base e $(+$ en $)\}$ (dissimilation).

2) que l'objet de sá $\mathrm{du}_{11} / \mathrm{e} / \mathrm{di}$ peut être à l'abs. (calque de l'acc. kašädu), ce que toutefois OBGT IX 55 sqq. ne semble pas confirmer (datif?).

L'autre possibilité serait de voir en -e- le "préfixe datif», 2ème ps. sg., que j'ai brièvement discuté en ZA 74, 41 sq. $^{42}$

\section{NBGT}

35. I 47-70 (cf. Black, o.c. 89 sqq.):

\begin{tabular}{|c|c|c|}
\hline 47-53 & $\mathrm{un} / \mathrm{an} / \mathrm{in} / \mathrm{en} \mathrm{ab} \mathrm{mu} \mathrm{u} \mathrm{u}^{43}$ & $a-n a-k u$ ma-lu-ú AN.TA MURU 4 .TA \\
\hline 54-58 & $\begin{array}{l}\text { un/an/in/en mu } \\
\text { (e.g.: *in-bùlug } \cdot e^{44}, \grave{i}-t u-d e ̀-\end{array}$ & $\begin{array}{l}j a-a-t i \text { AN.TA KI.TA } \text { MURU }_{4} \cdot \mathrm{TA} \\
\left.\mathrm{n}, \mathrm{mu}-\mathrm{un} \cdot \mathrm{du}_{8} \cdot \mathrm{e}\right)\end{array}$ \\
\hline & $\begin{array}{l}\text { un/an/in/en ab u } u_{8} \\
\text { (e.g.: ba-du-un) }\end{array}$ & at-ta ma-lu-ú KI.TA \\
\hline-70 & $\begin{array}{l}\text { un/an/in/en ab u } \\
\text { (e.g.: mu-un-du } u_{0}-\text { e } / \text { en }\end{array}$ & $\begin{array}{l}k a-a-t a M_{M R U_{4} \cdot T A} \text { KI.TA } \\
\left.: m u-e-d u_{8}-e !\right), i-t u-d e ̀-\epsilon\end{array}$ \\
\hline
\end{tabular}

42 Supprimer la référence à $\mathrm{ELA}_{2} 94$.

${ }^{43}$ Dans les lignes qui suivent, je ne discute pas les éléments ab, $m u$ et $u_{8}$.

$44=$ sil m'élèvew; type non attesté. 
Cf. II 95-102:

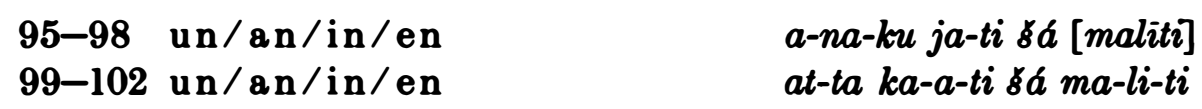

Mis a part le fait que la distinction constatée dans les textes paléobab. entre les allographes de l'abs. de la lère et de la 2ème ps. sg. n'est pas observée, les ll. 54-70 semblent confirmer l'hypothèse de la préfixation possible des éléments en question. Les 11. 47-53 (attendu: KI.TA) semblent corrompues.

\section{Interprétation des données au niveau morphématique.}

Si la détermination des allographes des préfixes abs. de la lère et de la 2ème ps. sg. dans les bases marû est chose relativement aisée, l'interprétation de la structure morphématique sous-jacente soulève, en revanche, des problèmes considérables. L'alternative est la suivante:

- Soit le morphème de l'abs. de la lère sg. differe de celui de la 2ème. Cette hypothèse ne peut être à priori exclue; elle implique toutefois que les morphèmes de l'abs. de la lère/2ème sg. ne sont pas identiques dans les bases hamţu ( $\{e n\})$ et maru $\left(\left\{\mathbf{x}_{1} / \mathbf{x}_{2}\right\}\right)$.

- Soit le morphème de l'abs. de la lère/2ème sg. est \{en\} (= "sujet" lère/2ème ps. sg. des verbes non ergatifs ${ }^{45}$ ). Cela s'harmonise parfaitement avec la structure ergative de la langue; la distinction entre les allographes de la lère et de la 2ème ps. sg. dans les bases marî reste toutefois à justifier.

En faveur de cette seconde hypothèse, il convient de noter:

1) qu'avant l'époque paléobab., la distinction entre l'abs. de la lère et de la 2ème sg. n'est pas explicitée graphiquement. Les exemples sont toutefois trop peu nombreux pour permettre de tirer des conclusions générales.

2) que si mon interprétation des NP Ha-ba/ma(-an)-zi-zi (ex. 18) est correcte, l'allographe de la 2ème sg. est, à l'époque d'Ur III, - a $n$ - après [a] (versus -e-dans les bases marû à l'époque paléobab.).

45 C'est aussi l'opinion de van Dijk, cf. ci-dessus n. 5. 
3) qu'à l'époque paléobab., l'allographe de la 2ème sg. est - i $n$ après [i] (séquence - Ci-e- + base normalement évitée ${ }^{46}$ ), -e $n$ après ḩé- en Lugal-e 577, $\mathrm{Y}_{3}+\mathrm{E}_{5}$ (ex. 31).

4) que dans un cas enfin (Lugal-e $540=$ ex. 22), la séquence me-e $n$ - est attestée.

Il est, en conséquence, plausible d'admettre:

1) que la distinction entre les allographes de l'abs. de la lère et de la 2ème ps. sg. dans les bases marû est un phénomène de date paléobab. A partir de cette époque, -e -, senti comme le morphème typique de la 2 ème ps. sg., a été préféré a -Vn-pour expliciter l'abs. de la 2ème sg. après [a] et [u].

2) que, jusqu'à l'époque paléobab., l'allophone de $\{e n\}$ (abs. lère/2ème sg.) dans les bases marî était, dans tous les environnements, [n]. A cette date, une distinction s'opère entre l'allophone de la lère sg. ([n]) et ceux de la 2ème sg ([e(n)] après [u] et [a], [n] après [i] et [e]). Que les graphies du type mu - e- ne sont pas seulement morphophonologiques, mais recouvrent une réalité phonétique, est assuré par les nombreuses occurences de me(-e) - .

\section{Conclusion.}

Rappelons brièvement les principaux résultats obtenus:

- Le morphème de l'absolutif de la lère et de la 2ème sg. est \{en\}, préfixé dans les bases marû, suffixé dans les bases hamtu.

- Avant l'époque paléobab., $\{e n\}(=/ n /)$ n'est, à de rares exceptions près (ex. 18), pas explicitement noté dans les bases marû, mais peut être implicitement marqué par l'emploi des allomorphes $/ \mathbf{n i} / \mathrm{de}\{\mathbf{n}\}$ et $/ \mathrm{mu} / \mathrm{de}\{\mathrm{m}\}$.

- A l'époque paléobab., une distinction est opérée, dans les bases marâ après [u] et [a], entre l'abs. de la lère ps. sg. $(/ \mathbf{n} /)$ et celui de la 2ème $(/ e(n) /)$.

${ }^{46}$ Cf. la séquence préfixale -e-ni- (néologisme de la fin(?) de l'époque paléobab.), issue probablement du besoin d'expliciter (orthographiquement!) l'ergatif de la 2ème ps. 8g. après le préf. loc. $\{n\}$. L'emploi toutefois de l'allomorphe /ni/ (au lieu de /n/) me semble indiquer le caractère secondaire de cette forme. 